\title{
Trajectory planning of rescue robot based on TRIZ effect
}

\author{
LIU Hong-liang*, YANG Li-song,TAN Hai-dong, CHEN Li-yan
}

Changchun University of Technology, Changchun, China; 130012

Key words: Trajectory planning, TRIZ, effect

ABSTRACT:In this paper, based on the TRIZ effect, combined with the working mechanism of the rescue robot, determine the parameters affecting the working performance of the rescue robot. And through the principle of conflict resolution, find the principle of the invention, Trajectory planning for rescue robot,and provide reference for optimal design of mechanical structure and control system of robot.

\section{Introduction}

As an important branch of the robot field, the rescue robot is always a hot and difficult research topic in the field of robotics because of the complexity of its structure and the diversity of its functions. The function of the rescue robot is finally reflected by its end effector. The reasonable planning of the running track of the end effector and the robot manipulator plays an important role in the operation efficiency and the ability of the robot to avoid obstacles.

Based on the theory of inventive problem solving, through the study on the working mechanism of the rescue robot, find the robot in the running process to improve the parameters and deterioration of parameters, and further to find out the principle of the invention has a greater contribution to solving technical problems, and in accordance with the principle of trajectory planning method for robot manipulator is designed, by some calculations, to calculate the parameters of the process, draw the trajectory. This method can effectively carry out the planning and design of the robot arm and the end effector trajectory, provide an important reference for the design of mechanical structure and control system of the robot, shorten the product development cycle, improve the efficiency of product development. ${ }^{[1,2,3]}$

\section{Theory of inventive problem solving}

\subsection{Technology evolution method}

If users know their products is the stage of the life cycle (infancy, growth stage, mature stage and exit stage, then solve the stage) list of modules that can be used to solve the problem, a problem using TRIZ technology evolution mode and route design. The evolutionary model, evolutionary route and the combination of a large number of engineering examples into an evolutionary tree, there are four directories in the evolutionary tree, the evolutionary model is a directory, the evolution route is two level directory, evolutionary state and evolution is tied to the three level directory, four level directory instance is improved, specifically for the user to solve the engineering problems. ${ }^{[2,3,4]}$

\subsection{Evolution model}

Law 1: the law of the integrity of the system. A manipulator system must be composed of four parts, namely, energy device, joint motor, transmission parts, control devices, as shown in the following figure 
Law 2: the law of energy transfer.

Law 3: the law of harmony of alternating motion.

Law 4: to increase the level of the law of the ideal.

Law 5: the unbalanced development law of parts and components.

Law 6: the law of transfer to the super system.

Law 7: the macro - to micro - law of transfer.

Law 8: increase the law of the integrity of matter field.

\section{The concept of TRIZ effect}

Single mode: an effect can be achieved with different functions, in the hands of the serial manipulator, changes in length can be changed at the end of the speed and position. But in many designs, a function requires multiple effects, and these effects can be composed of the association effect model or the control effect model.

Association effect mode: the mode of association effect refers to the effect of the combination of several effects in order to achieve a function, such as the double gold chip used to rely on the thermal expansion effect and the Hooke effect. It consists of series, parallel and parallel ring and several basic forms.

The following figure is a block diagram of several main association effects.

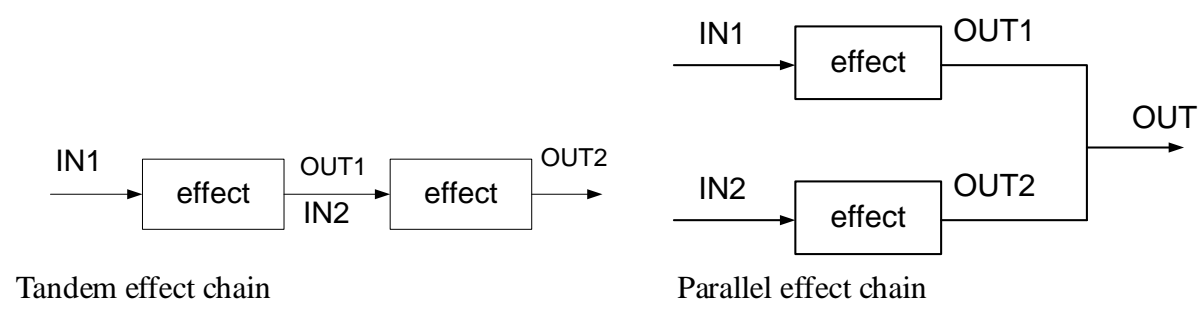

Figure 1 Series effect and parallel effect

In this system, according to the specific requirements of the manipulator, the effect of chain should be designed by at least 5 single mode consisting of series effect chain, when necessary, in order to meet the stability of the manipulator's end, but also can increase the chain parallel effect necessary.

\section{Trajectory planning of manipulator}

Robot trajectory is the specific parameters of the acceleration, velocity and position of all degrees of freedom in the robot motion. Through a certain calculation method, the process of calculating these parameters is called trajectory planning.

The Archie Schuler contradiction matrix, the following two parameters,

To improve the parameters: the length of the stationary object (3), the speed (9), and the force (10)

The deterioration of the parameters: operability (33), adaptability and multi use (35)

By searching the conflict table, from 40 in 17 to find the principle of invention invention principle to solve the technical problems, among them, three contributions are: the principle of maximum dimension change (17), the potential of (12) and reverse (13).

In this paper, the three principles of the invention are used to track the trajectory of the end position of the rescue robot. 
With the change of dimension, the number of the connecting rod can be changed in series with the mechanical hand, and the potential can be understood as the different attitude of the end effector to the same position, that is, the different solutions. These two are the important factors for the trajectory planning of the end position.

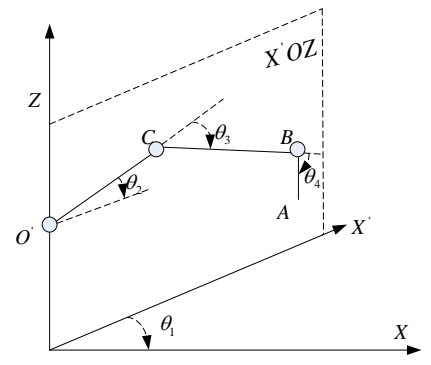

Figure 2 Schematic diagram of robot in Cartesian space

It is assumed that the motion trajectory of the robot gripper mechanism is a straight line $\mathrm{AB}$. The average $\mathrm{AB}$ is divided into 5 equal parts, calculated as the figure and the value of the process, that is, the interpolation between the straight line. Although the motion trajectory is a straight line, the amount of the joint needs to be calculated. Path segmentation should be appropriate, otherwise the trajectory will appear irregular. In order to make motion for a straight line, the segmentation points as much as possible, at the same time, the corresponding increase in joint points, the calculation is also relatively large.

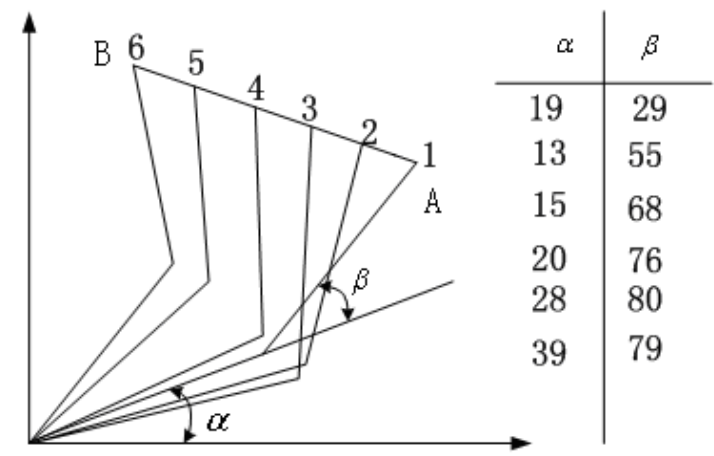

Figure 3 Motion of 3 and 4 degrees of freedom in Cartesian space of micro robot manipulator

In order to ensure the smooth motion of the robot, the trajectory function is expressed by the following five Polynomials:

$$
s(t)=a_{0}+a_{1} t+a_{2} t^{2}+a_{3} t^{3}+a_{4} t^{4}+a_{5} t^{5} \quad t \in\left(t_{0}, t_{f}\right)
$$

As the motion trajectory for a straight line, there must be accelerated, uniform, slow three stages. $S_{0}$ is the initial point displacement of acceleration and deceleration motion, and $S_{f}$ is the movement track.

(1) Trajectory planning of accelerated motion in Cartesian space

The initial condition of the acceleration segment is set to:

$$
\begin{array}{lcc}
s\left(t_{0}\right)=s_{0}, & s\left(t_{f}\right)=s_{f} & \left(\text { End device displacement from } s_{0} \text { to } s_{f}\right) ; \\
s^{\prime}(t)=0, & s^{\prime}\left(t_{f}\right)=v_{f} & \left(\text { velocity increase from0 to } v_{f}\right) ; \\
s^{\prime \prime}\left(t_{0}\right)=0, & s \theta t(t)=0 & \text { Initial angular acceleration and Final angular acceleration }
\end{array}
$$




$$
\begin{gathered}
\operatorname{are} 0) .\left(t_{0}=0\right) \\
\mathrm{t} \in\left[t_{0}, t_{f}\right]
\end{gathered}
$$

Can launch:

$$
\left\{\begin{array}{l}
a_{0}=s 0 ; a_{1}=0 ; a_{2}=0 \\
a_{3}=\left[10\left(s_{f}-s_{0}\right)-4 v_{f} t_{f}\right] / t_{f}^{3} \\
a_{4}=\left[7 v_{0} t_{f}-15\left(s f-s_{0}\right)\right] / t_{f}^{4} \\
a_{5}=\left[6\left(s_{f}-s_{0}\right)-3 v_{f} t_{f}\right] / t_{f}^{5}
\end{array}\right.
$$

It will be substituted into the formula 1, get the equation of trajectory planning of the acceleration section.

Deceleration motion trajectory planning in Cartesian space

The initial conditions in the deceleration section can be set to

$$
\begin{aligned}
s\left(t_{0}\right) & =s_{0}, & s\left(t_{f}\right)=s_{f} & & \left(\text { End device displacement from } s_{0} \text { to } s_{f}\right) ; \\
s^{\prime}(t) & =v_{0}, & s^{\prime}\left(t_{f}\right)=0 & & \left(\text { velocity reduce from } v_{0} \text { to } 0\right) ; \\
s^{\prime \prime}\left(t_{0}\right) & =0, & s \theta \text { t t })=0 & & \text { (Initial angular acceleration and Final angular acceleration are }
\end{aligned}
$$

$0) .\left(t_{0}=0\right)$

Can launch:

$$
\left\{\begin{array}{l}
a_{0}=s_{0} ; a_{1}=v_{0} ; a_{2}=0 \\
a_{3}=\left[10\left(s_{f}-s_{0}\right)-6 v_{0} t_{f}\right] / t_{f}^{3} \\
a_{4}=\left[8 v_{0} t_{f}-15\left(s_{f}-s_{0}\right)\right] / t_{f}^{4} \\
a_{5}=\left[6\left(s_{f}-s_{0}\right)-3 v_{0} t_{f}\right] / t_{f}^{5}
\end{array}\right.
$$

The formula in equation 2 , can be obtained at the end of the deceleration trajectory equation.

Uniform motion trajectory planning in Cartesian space:

While $\mathrm{t} \in\left[t_{0}, t_{f}\right]$, The end track uniform motion can be expressed as below:

$$
s(t)=s_{0}+v t
$$

Among them, $\mathrm{v}$ is the uniform velocity; the $s_{0}$ is the initial displacement of $t_{0}$.

From the calculation, it is easy to see that the trajectory planning method of the Cartesian space, the trajectory of the motion of the uniform segment is a time proportional function. At the end of the constant speed, acceleration is zero, the movement form is relatively simple, but the actual movement process, end track by means of rotating trajectory control and multi joint realization of trajectory itself, the need for solving the inverse kinematics problem, this is not much discussion. ${ }^{[4,5]}$

\section{Conclusion}

Based on the theory of inventive problem solving, combined with the TRIZ effect, the working performance of the manipulator parameters to find the impact of the rescue robot, combined with 
the technology of conflict resolution principle, selection principle of invention right, and further carry out the robot trajectory planning, provide a reference for the optimization design of the mechanical structure and control system of the robot.

\section{Acknowledgement}

This work was financially supported by the Jilin province education department Twelfth Five-Year Scientific research project (No.[2013]153).

\section{Reference}

[1] ZHANG Tiantian.TAN Yufei.ZHANG Xuedan.et al. A novel hybrid technique for leak detection and location in straight pipelines[J]. Journal of Loss Prevention in the Process Industries.2015.35: 157-168.

[2] ZHAN Xiang-hui,LI Yan,JIA Ai-jun,SUN Yu-shuai.. Scientific effects base for creative design [J]. JOURNAL OF ENGINEERING DESIGN. 2005, 12(1).

[3] RAY KIRBY J,INDER WADEHRA. Designing business machines for disassembly and recycling[A].Arlington,Virginia 1993.

[4] LIU Zhifeng,HU Di,GAO Yang,JIANG Hao. TRIZ Based Revised Design for Disassembly of Joint Structure [J]. JOURNAL OF MECHANICAL ENGINEERING. 2012, 48(11)

[5] Sung-Uk Lee,Pyung Hun Chang. Control of a heavy-duty robotic excavator using time delay control with integral sliding surface[J].Control engineering practice 2002,7(7) 